\title{
The 3D Smith chart from theory to experimental reality check
}

Andrei A. Muller ${ }^{1}$, Victor Asavei ${ }^{2}$, Alin Moldoveanu ${ }^{2}$, Esther-Sanabria-Codesal ${ }^{3}$, Riyaz A. Khadar $^{4}$, Cornel Popescu ${ }^{2}$, Dan Dascalu ${ }^{5}$, Adrian. M. Ionescu ${ }^{1}$

1 Nanoelectronic Devices Laboratory (NanoLab), École Polytechnique Fédérale de Lausanne (EPFL), 1015, Lausanne, Switzerland,

${ }^{2}$ Department of Computer Science and Engineering, Faculty of Automatic Control and Computers, University Politehnica of Bucharest, 060042 Bucharest, Romania,

${ }^{3}$ Departamento de Matemática Aplicada, Universitat Politècnica de València ,46022 Valencia, Spain,

${ }^{4}$ Powerlab, École Polytechnique Fédérale de Lausanne (EPFL), 1015, Lausanne, Switzerland.

${ }^{5}$ Faculty of Electronics, University Politehnica of Bucharest, 061071 Bucharest, Romania.

-accepted article, -IEEE Microwave Magazine, vol 21, no 11, pp 22-35, Nov. 2020 (unedited)

\section{History of the Smith chart}

The Smith chart was primarily developed, extended, and refined by Phillip Hagar Smith [1], in a series of published works [2-4] between 1939 and 1969.

Phillip Smith was born in 1905 in Lexington, Massachusetts. He majored in electrical communications at Tufts University and joined the Radio Research Department of Bell Telephone Laboratories in 1928.

Around 1930, while employed at Bell Telephone Laboratories, Smith started his work on the diagram that was to become the Smith chart. He submitted the initial version of his diagram to Electronics Magazine in 1937; the magazine finally published it in 1939 [2]. In 1940 the M.I.T. Radiation Laboratory started to use the chart. In 1944 Smith published a second article which incorporated further improvements, including the use of the chart with either impedance or admittance coordinates. In 1952 Smith became an IEEE Fellow for his contributions to the development of antennas and graphical analysis of transmission line characteristics. In 1958, in the first issue of Microwave Journal, a biography of Smith was published to acknowledge the importance of his contributions. In 1969 he wrote the book Electronic Applications of the Smith Chart in Waveguide, Circuit and Component Analysis; he retired from Bell Labs in 1970. In 1975 he received the MTT Special Recognition Microwave Application Award for the Smith chart. In 1994 he was elected to the New Jersey Inventors Hall of Fame.

In this article, we start with several drawings of spherical Smith charts, proposed with the aim of having a generalized Smith chart that deals with both negative and positive resistance circuits within a compact surface. Then, we introduce our suggested 3D Smith chart, discussing the Smith chart main equation from an inversive geometry perspective, for which Mobius transformations are a simple type of transformation, mapping circles into circles on a special sphere (called a Riemann sphere [5]), leading to mathematical completeness and simplicity while dealing with negative resistance and infinity. In order to familiarize the reader with the notions of dynamics and oriented curvature used further on to enhance the 3D Smith chart and to analyze negative capacitance and inductance, the equation of the unit circle is presented from a clockwise-counterclockwise dynamical viewpoint, mirroring the reflection coefficient of a positive/negative capacitance.

The construction of the proposed 3D Smith chart is briefly presented, including the main properties and critical points and its analogy with the earth/latitude/longitude perspective, as well as its advantages and drawbacks.

Then, to acclimate the reader to the earth-like chart, several matching examples are given on both Smith and 3D Smith charts in a parallel one-to-one comparison. The use of 3D space for displaying several scalar frequency dependent parameters is described.

The 3D Smith chart's practical benefits are described for passive circuits using inductor design and then for active amplifier stabilization and power gain problems. Last, negative capacitance analysis and detection is presented on the 3D Smith chart followed by analysis of a simple negative resistance tunnel diode equivalent circuit. 
The article takes the reader on a journey to a compact tool where negative and positive resistance are all analyzed on a preserving-circles patterned sphere. We propose to use the 3D space surrounding the sphere for multiple complex-scalar visualization, which is useful in equivalent circuit extractions, multi-parameter optimization, or simply for multiple-variable imaging and for uncovering of changes in frequency orientation.

\section{Spherical Smith chart drawings for negative resistance circuits}

The Smith chart is limited within the unit circle to passive circuits with positive resistance $(r$ ) (or conductance $(g)$ ) [1]; circuits with negative $r$ (or $g$ ), which occurs in active circuits, are not covered by the conventional Smith chart $[4,6]$.

In 2006, IEEE Microwave Magazine presented an article [7] on drawings by the artist M. C. Escher, including a spherical self-portrait. The article pointed out the connection between the drawings of Escher and hyperbolic geometry while also emphasizing the connection between the Smith chart and Mobius transformations in geometry within the $2 \mathrm{D}$ complex plane.

Motivated by the desire to have a unified chart for both active and passive microwave circuits, Zelley proposed in [8] an intuitive spherical Smith chart drawing on a ping-pong ball. The drawing in [8] was still lacking "mathematical rigor" as the author acknowledges, with no equations given for its construction. Inspired by the beautiful drawing in [8], obtained by means of skillful but complicated arithmetical and trigonometrical manipulations, spherical Smith chart theories were proposed in [9-10]. In [9] the circles appearing in 2D are mapped into different curves on the spherical chart while in [10] the circles on the spherical Smith chart are distorted into ellipses in 2D.

Actually, in [9], the authors parameterize the sphere using two angles as parameters, so that the circles of resistance appear when we view the plane containing the equator from the point $(1,0,0)$ and the circles of constant reactance are displayed when we turn this plane sideways. With this structure, the orthogonal projection of these circles onto the equator plane (2D Smith chart), distorts them into ellipses.

In the same way, the curves on the sphere considered in [10] are projected orthogonally in 2D and do not have a circular shape, in general.

Here we will develop our main (initial) work on a single concept: inversive geometry. Inversive geometry considers the space of inversive transformations, which map all the circles into circles on a $2 \mathrm{D}$ sheet or on the Riemann sphere, when points are thrown to infinity $(\infty)$. For this geometry, $\infty$ is just a point [5, 11-13], unlike Euclidean geometry where $\infty=$ unending or hyperbolic geometry where $\infty=$ circle [7].

\section{D Smith chart and negative resistance}

In [12] we propose a single equation that maps the entire reflection coefficient $(\rho)$ (1) plane on a unit sphere $(s)$. The equation can be expressed in terms of $\rho$, (its real $\rho_{\text {r }}$ and imaginary $\rho_{\text {r }}$ part), normalized impedance $(z)$, resistance $(r)$, and reactance $(x)$ as follows (2).

$$
\begin{gathered}
\rho(z)=\frac{z-1}{z+1} \\
\rho_{3 D}\left(\rho=\rho_{r}+j \rho_{i}\right)=\left(\frac{2 \rho_{r}}{1+|\rho|^{2}}, \frac{2 \rho_{i}}{1+|\rho|^{2}}, \frac{1-|\rho|^{2}}{1+|\rho|^{2}}\right), \rho_{3 D}(z=r+j x)=\left(\frac{|z|^{2}-1}{|z|^{2}+1}, \frac{2 x}{|z|^{2}+1}, \frac{2 r}{|z|^{2}+1}\right)
\end{gathered}
$$

This guarantees the invariance of the circles and of the angles; the 3D Smith chart includes both active and passive microwave circuits on a single sphere, mapping the original Smith chart onto the northern hemisphere, and the circles which tend to infinity on the 2D extended Smith chart into circles in the southern hemisphere. Thus, the northern hemisphere contains circuits with positive resistance and the southern hemisphere contains circuits with negative resistance, east for inductive, west for capacitive.

In further developments of the concept [14-15], the 3D space surrounding the sphere is then used for visualizing a variety of parameters. Fig.1 shows the conventional Smith chart and 3D Smith chart in a direct comparison. Infinity or reflection coefficients with very high magnitudes are scattered in all 
directions on the 2D Smith chart (reflection coefficients of loads with negative resistance). On the 3D Smith chart, surpassing the unit magnitude of the reflection coefficient means simply moving south while infinity is a point on the sphere (south pole in geographical language).

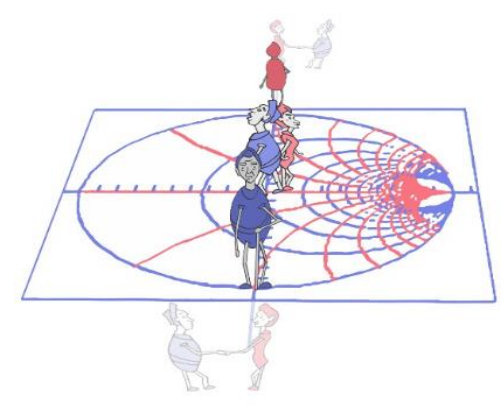

(a)

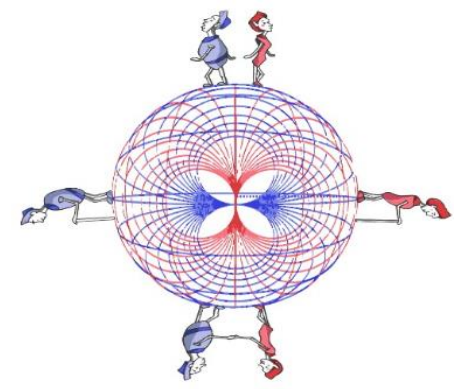

(b)

Fig. 1 A vision of infinity: (a) Moving away from the origin of the Smith chart (b) Moving away from the origin of the 3D Smith chart. The males and females are in one-to-one correspondence on the Smith chart and 3D Smith chart.

\section{Smith chart leading equation and negative resistance}

The leading equation that generates the Smith chart that is given in (1) is presented by Smith as a bilinear conformal transformation in [4]; however, nothing about the global geometrical properties of these transformations is disclosed in [2-4].

The equation maps the constant $r$ and constant $x$ grid lines of the right half plane $(r>0)$ of the impedance plane within the unit circle of the Smith chart into arcs and circles, leaving the left half plane $(\mathrm{r}<0)$ to be mapped in its exterior, as depicted in Fig. 2, while throwing points to infinity in all directions. Unlike the classical Smith chart literature which considers (1) a bilinear transformation $[4,7,16]$ or a conformal one [17-18], here we see it as a particular case of inversive transformation: the direct inverse: $D(z)$ (3) (a) [11]. Direct inversive (also called Mobius) and indirect $I(z)$ inversive transformations (3) (b) are the only transformations which map all generalized circles (simple circles or infinite extended lines) into generalized circles irrespective of their position on the complex plane. When points are thrown to infinity, one needs to compress the complex plane $\mathbb{C} \cup\{\infty\}$ Riemann sphere) in order to visualize them [5].

$$
D(z)=\frac{a z+b}{c z+d}(\mathrm{a}) ; I(z)=\frac{a \bar{z}+b}{c \bar{z}+d}(\mathrm{~b})
$$

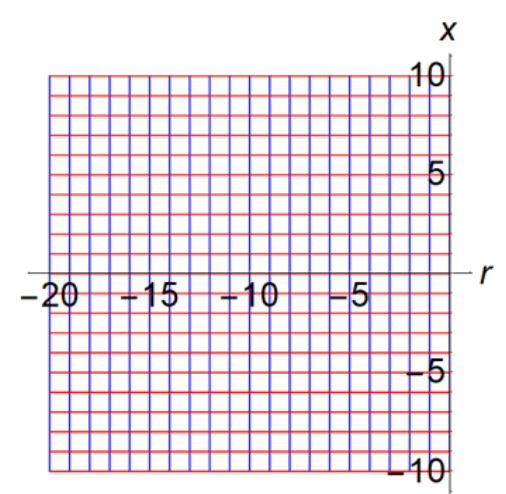

(a)

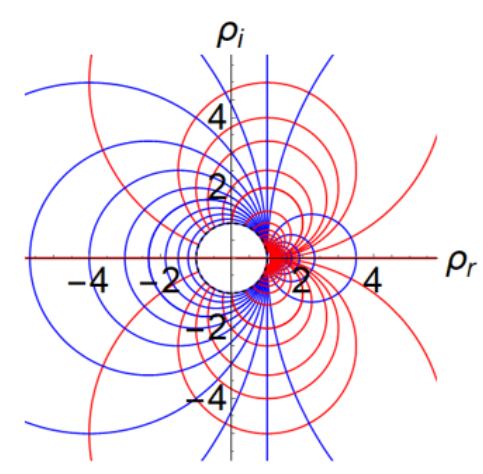

(b)

Fig. 2 The left half plane (LHP) of the impedance plane is mapped onto the exterior of the Smith chart. The constant $r$ circles (for negative r) are now mapped into circles outside of the Smith chart, while the constant $x$ circles are mapped into arcs outside of the Smith chart.

\section{Smith chart circle equation and negative capacitance and inductance}

In order to visualize circuits exhibiting clockwise and counterclockwise frequency orientation, the dynamics of the frequency path need to be grasped. There are a variety of circuits which exhibit reflection 
coefficient movements on the unit circle of the complex plane; however, their intrinsic nature can be very different. Table I presents different frequency parametrizations of the unit circle, with their angular frequency speed and specific orientation. The oriented curvature $(\mathrm{k}(\omega))$ gives the orientation of any possible circuit. Computing the reflection coefficient for a positive inductor or negative capacitor (or viceversa), one will see the identical circle-like path in the reflection plane. However, computing their curvature $k$ [19], one may spot sign changes and, in this case, find their different intrinsic natures and orientations. It can be seen in Table I that the reflection coefficient of a single-port normalized-positive capacitance is a direct inversive transformation (3) (a) of $z=1 /(j \omega C)$, while the reflection coefficient of the negative capacitance is an indirect inversive transformation (3) (b) of $z=1 /(j \omega C$ ), both of which have the same circle path, but opposed orientations. Later in this article we will show how the 3D Smith chart can spot orientation changes.

Table I: Different Perspectives on the Unit Plane Circle Depending on the Mathematical Viewpoint

\begin{tabular}{|c|c|c|c|c|}
\hline Viewpoint & Equation & Speed & $\begin{array}{l}\text { Orientation, } \\
\text { Curvature } \\
\text { (k) }\end{array}$ & $\begin{array}{ll}\text { Position at } & \omega=\pi / 2, \\
\text { (starting point } 1,0) & \end{array}$ \\
\hline $\begin{array}{l}\text { Algebraic, } \\
\text { 2D Euclidean } \\
\text { geometry }\end{array}$ & $r^{2}+x^{2}=1$ & No info & No info & No info \\
\hline $\begin{array}{l}\text { Differential } \\
\text { geometry }\end{array}$ & $\begin{array}{l}\operatorname{Cos}(\omega)+j \operatorname{Sin}(\omega) \\
\omega=\left\{0,2^{*} \pi\right.\end{array}$ & 1 & $\begin{array}{l}\text { Counter- } \\
\text { clockwise, } \\
\mathrm{k}=1\end{array}$ & $\begin{array}{c}\Gamma_{r} \\
1.06 \\
0.5 \\
-1.0-0.5 \\
-0.5 \\
-1.0\end{array}$ \\
\hline $\begin{array}{l}\text { Differential } \\
\text { geometry }\end{array}$ & $\begin{array}{l}\operatorname{Cos}(-2 \omega)+\mathrm{j} \operatorname{Sin}(-2 \omega) \\
\omega=\left\{0,2 *_{\pi}\right\}\end{array}$ & 2 & $\begin{array}{l}\text { Clockwise, } \\
\mathrm{k}=-1\end{array}$ & $\begin{array}{c}\Gamma_{r} \\
1.0^{0.5} \\
-1.0-0.5 \\
-0.5 \\
-1.0\end{array} \quad 0.5 \quad 1.0^{\Gamma_{i}}$ \\
\hline $\begin{array}{l}\text { Reflection } \\
\text { coefficient } \\
\text { positive } \\
\text { capacitor }\end{array}$ & $\begin{array}{r}\rho=\frac{\frac{1}{j \omega C}-1}{\frac{1}{j \omega C}+1} \\
\omega=\{-\infty, \infty\}\end{array}$ & $\frac{2 C}{1+C^{2} \omega^{2}}$ & $\begin{array}{l}\text { Clockwise, } \\
\mathrm{k}=-1\end{array}$ & forC=1 $\begin{array}{c}\rho_{i} \\
1.0^{1} \\
0.5 \\
-1.00 .5 \\
-0.5 \\
-9.0\end{array}$ \\
\hline $\begin{array}{l}\text { Reflection } \\
\text { coefficient } \\
\text { negative } \\
\text { capacitor }\end{array}$ & $\begin{array}{l}\rho=\frac{-\frac{1}{j \omega C}-1}{-\frac{1}{j \omega C}+1} \\
\omega=\{-\infty, \infty\}\end{array}$ & $\frac{2 C}{1+C^{2} \omega^{2}}$ & $\begin{array}{l}\text { Counter- } \\
\text { Clockwise, } \\
\text { k=1 }\end{array}$ & forC $=1 \quad \begin{array}{c}\rho_{i} \\
.1 .0 \\
0.5 \\
-1.0-0.5 \\
-0.5 \\
-1.0\end{array}$ \\
\hline
\end{tabular}

\section{D Smith chart construction}

Fig. 3 shows the construction of the 3D Smith chart using stereographic projection from the south pole with its guiding equation $(2)(\mathbb{C} \cup\{\infty\})$. The construction is based on the circles visualization theory of 
direct inversive and indirect inversive transformation (3), first proposed in 1914 by Maxime Bôcher, former president of the American Mathematical Society [5].

The classical Smith chart is mapped in the northern hemisphere (Fig.3 (a)); the circuits with negative resistance, exhibiting $\left.\rho_{\mid}\right|_{\mid}>1$, are mapped in the southern hemisphere in Fig. 3(b). The 3D chart's properties are listed in Table II. Fig. 4 presents the 3D Smith chart with its key points in a one-to-one comparison with the Smith chart and the earth. Table II lists its properties and Table III its possible advantages and drawbacks.

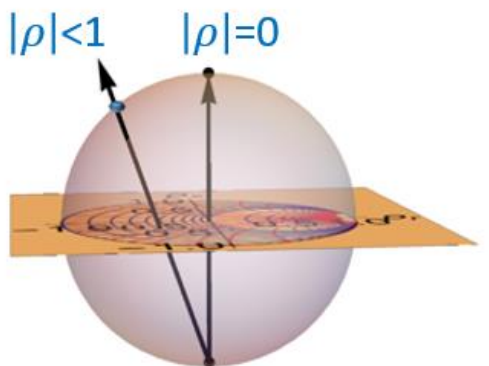

(a)

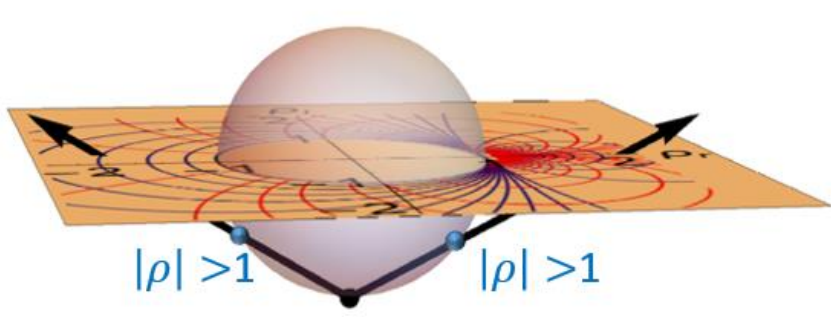

(b)

Fig. 3 3D Smith chart construction: (a) 3D Smith chart image of circuits with $|\rho|<1$, (b) $|\rho|>1$, southern hemisphere Table II: Properties of the 2D Smith Chart versus the 3D Smith Chart

\begin{tabular}{|c|c|c|}
\hline Property & 2D Smith Chart & $\begin{array}{l}\text { 3D Smith Chart } \\
\text { GCS Language (Latitude, Longitude) }\end{array}$ \\
\hline Positive resistance & Interior of unit circle & Northern hemisphere \\
\hline Negative resistance & Exterior & Southern hemisphere \\
\hline Perfect matching & Origin & North pole \\
\hline $\mid$ Reflection coefficient $\mid==$ infinity & Infinity, far out & South pole \\
\hline Inductive & Above $\Gamma_{\mathrm{r}}$ axes & East \\
\hline Capacitive & Below $\Gamma_{\mathrm{r}}$ axes & West \\
\hline $\mathrm{r}, \mathrm{x}, \mathrm{g}, \mathrm{b}$ constant & Circles & Circles \\
\hline Purely resistive & $\Gamma_{\mathrm{r}}$ axes & $\begin{array}{l}\text { Great circle formed by prime meridian and } \\
\text { antemeridian dividing the world into east } \\
\text { and west hemispheres }\end{array}$ \\
\hline$|\Gamma|=$ constant & Circle centred in the origin & Latitude circle \\
\hline $\begin{array}{l}\begin{array}{l}\text { Constant phase of the reflection } \\
\text { coefficient }\end{array} \\
\end{array}$ & Lines through the origin & $\begin{array}{l}\text { Great circle of longitude made by the prime } \\
\text { meridian and antemeridian }\end{array}$ \\
\hline$|\Gamma|=1$ & Unit circle & Equator \\
\hline Open circuit & $(1,0)$ & $\begin{array}{l}\text { Null island (prime meridian/equator } \\
\text { intersection) }(0,0)\end{array}$ \\
\hline Short circuit & $(-1,0)$ & $\begin{array}{l}\text { Antipodal point to null island (ante } \\
\text { meridian/equator intersection } \\
(0,-180 \mathrm{E})\end{array}$ \\
\hline Unit inductive load & $(0, \mathrm{j})$ & $(0,90 \mathrm{E})$ \\
\hline Unit capacitive load & $(0,-\mathrm{j})$ & $(0,90 \mathrm{~W})$ \\
\hline
\end{tabular}

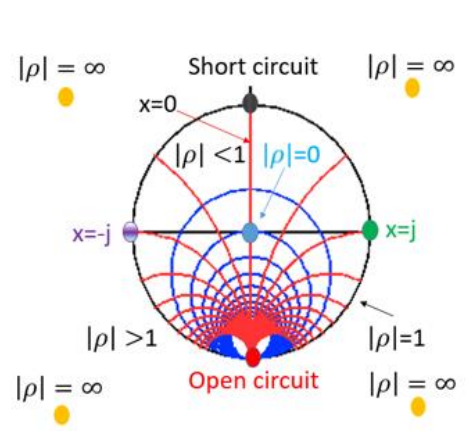

(a)

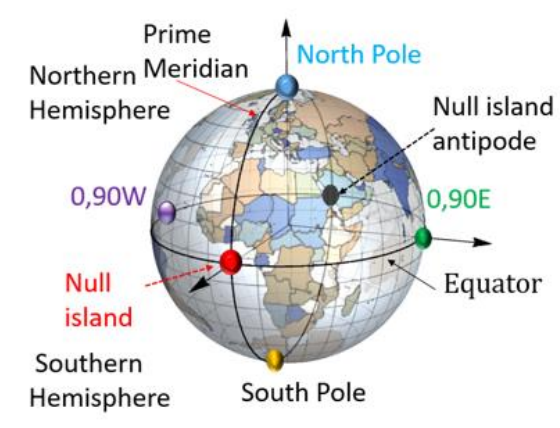

(b)

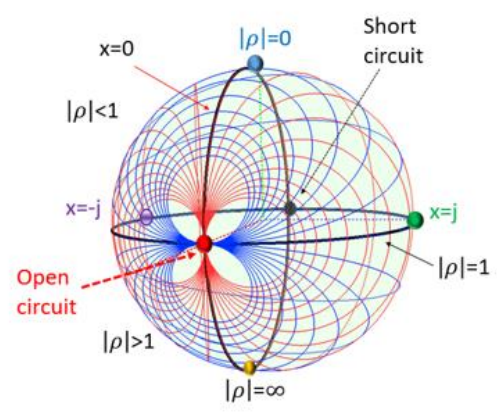

(c)

Fig. 4 (a) 2D Smith chart, (b) Earth and (c) 3D Smith chart rendered with $r$ (blue), $x$ circles (red) points and zones correspondence 
Fig. 5 represents the 3D Smith chart and Smith chart rendered with constant $r, x, g$, and $b$ (susceptance circles). The CAD tool in which it is (or may be) implemented allows the viewer to rotate it in any convenient way for the desired application.

Table III: Uses, Advantages, and Disadvantages of the 3D Smith Chart

\begin{tabular}{|c|c|c|c|}
\hline Use & Circuit Examples & Location & Advantages/Disadvantages \\
\hline $\begin{array}{l}\text { Negative resistances in [12- } \\
\text { 13] and in this paper }\end{array}$ & $\begin{array}{l}\text { In amplifier stability, } \\
\text { diodes, oscillators, } \\
\text { etc. }\end{array}$ & $\begin{array}{l}\text { South hemisphere } \\
\text { (surface) }\end{array}$ & $\begin{array}{l}\text { Compact space for all possible loads on a sphere } \\
\text { (thus a surface)/ CAD or a 3D printed chart } \\
\text { needed }\end{array}$ \\
\hline $\begin{array}{l}\text { Gain representations/group } \\
\text { delay in [14-15] and in this } \\
\text { paper }\end{array}$ & Amplifiers & $\begin{array}{l}\text { 3D space (exterior } \\
\text { when positive, interior } \\
\text { when negative) }\end{array}$ & $\begin{array}{l}\text { Scalar levels can be seen simultaneously with S- } \\
\text { parameters/ CAD chart needed }\end{array}$ \\
\hline $\begin{array}{l}\text { Frequency dynamics [19] } \\
\text { and in this paper }\end{array}$ & $\begin{array}{l}\text { All circuits, negative } \\
\text { capacitances, } \\
\text { negative inductances, } \\
\text { metamaterials }\end{array}$ & 3D space & $\begin{array}{l}\text { S-parameters clockwise, counterclockwise } \\
\text { changes can be spotted, intrinsic physical } \\
\text { connections can be made / CAD chart needed }\end{array}$ \\
\hline $\begin{array}{l}\text { Multiparameter in } \\
\text { optimizations/equivalent } \\
\text { circuit extractions in [19] } \\
\text { and in this paper }\end{array}$ & $\begin{array}{l}\text { In the } \\
\text { design/modelling of } \\
\text { all circuits }\end{array}$ & Surface, 3D space & $\begin{array}{l}\text { Simultaneously fit S-parameters \& orientation } \\
\text { inductances, Qs, fulfill multiple } \\
\text { requirements/correct extraction of equivalent } \\
\text { circuit / CAD chart needed }\end{array}$ \\
\hline
\end{tabular}

Table III lists the various properties of the Smith chart and 3D Smith chart. Fig. 5 shows the 3D Smith chart rendered with the constant normalized resistance, reactance(x), conductance $(\mathrm{g})$, and susceptance circles (b) in comparison with the Smith chart. The $r, g \in(0, \infty)$ for the Smith chart while $r, g \in(-\infty, \infty)$ for the 3D Smith chart.

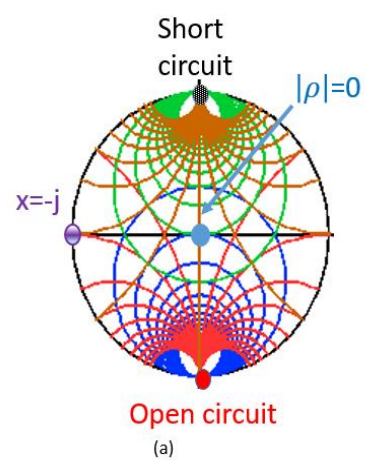

(a)

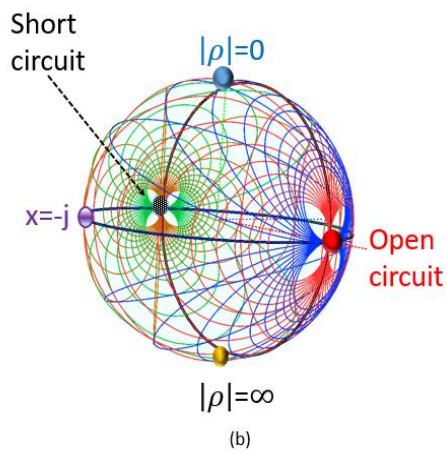

Fig.5 (a) Smith chart (b) and 3D Smith chart rendered with r,x,g,b circles

\section{Matching a network on the chart surface}

In order to match the desired network, one has to intersect the $r=1$ or $g=1$ circles which pass through the origin of the Smith chart in Fig. 6 (a) or through the north pole of the 3D Smith chart (Fig. 6 (b)). When the north pole is reached, matching is achieved. The matching procedure is identical to that used in $2 \mathrm{D}$; the designer simply has to move on the constant $r$ and constant $g$ circles or on the constant reflection coefficient circles (latitude) in order to intersect the $r=1$ and $g=1$ circles in the fashion desired. Fig 6. (c) presents a variety of matching strategies and their circuital meaning, while moving on the constant $r, g$ circles in opposed directions. On the 3D Smith chart, the orientation is best visualized as if the viewer were a person who is walking on the surface with their feet on the circles. 


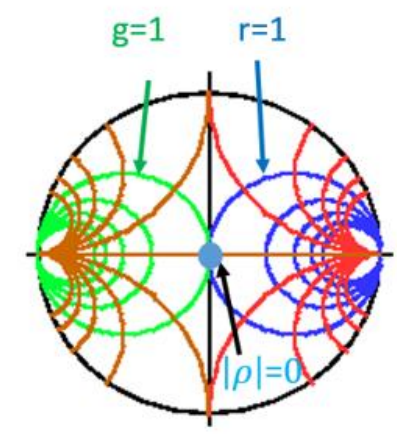

(a)

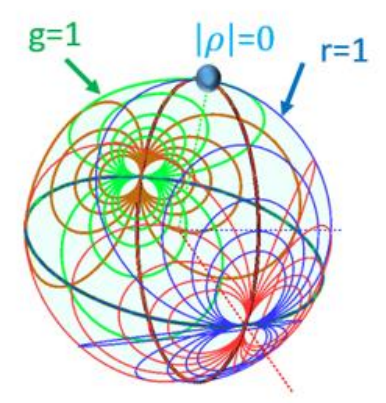

(b)
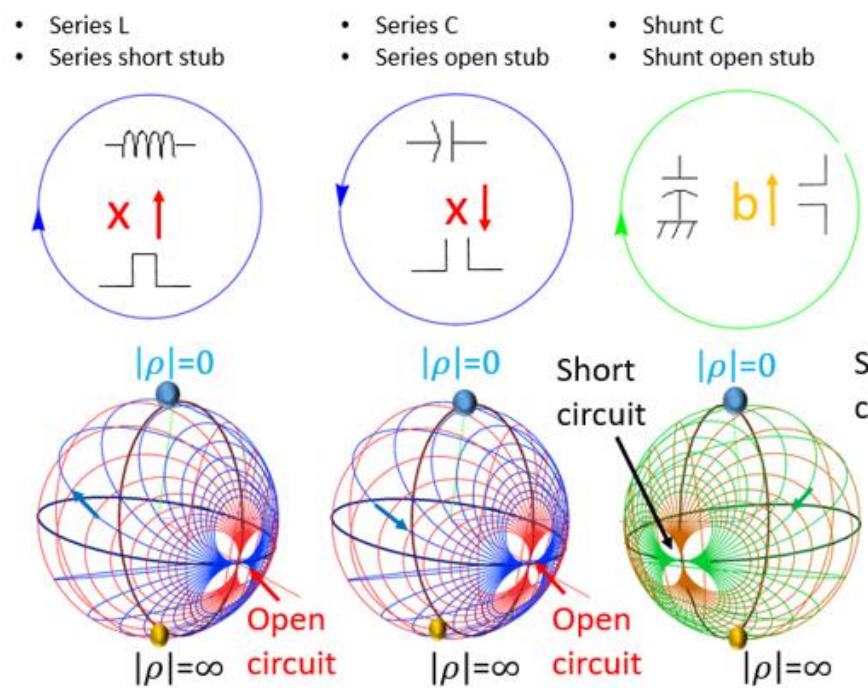

- Shunt L

- Shunt short stub

(c)

Fig. 6 Matching a network: (a) Smith chart $g=1, r=1$ circles, (b) $3 D$ Smith chart $g=1, r=1$ circles (c) Moving along $g$ and $r$ constant circles on the 3D Smith chart, clockwise and counterclockwise-circuital correspondence

\section{Example 1: matching a given passive network}

To match a load of $10+\mathrm{j} 10$ to a $50 \Omega$ line with $\mathrm{L}, \mathrm{C}$ networks: We normalize to $50 \Omega$ : and get $\mathrm{z}=0.2+\mathrm{j} 0.2$. Fig. 7 presents schematically the matching of a $z=0.2+0.2 j$ network with various circuits. The values of these elements can be easily computed as on the 2D Smith chart, keeping in mind the coordinates of the points and equation (2). Fig. 7 shows this: (a) with a series capacitance and shunt inductance, (b) series L and shunt $\mathrm{C}$, (c) lossless transmission line (moving on a latitude circle) and shunt short stub, and (d) lossless transmission line and shunt short stub.

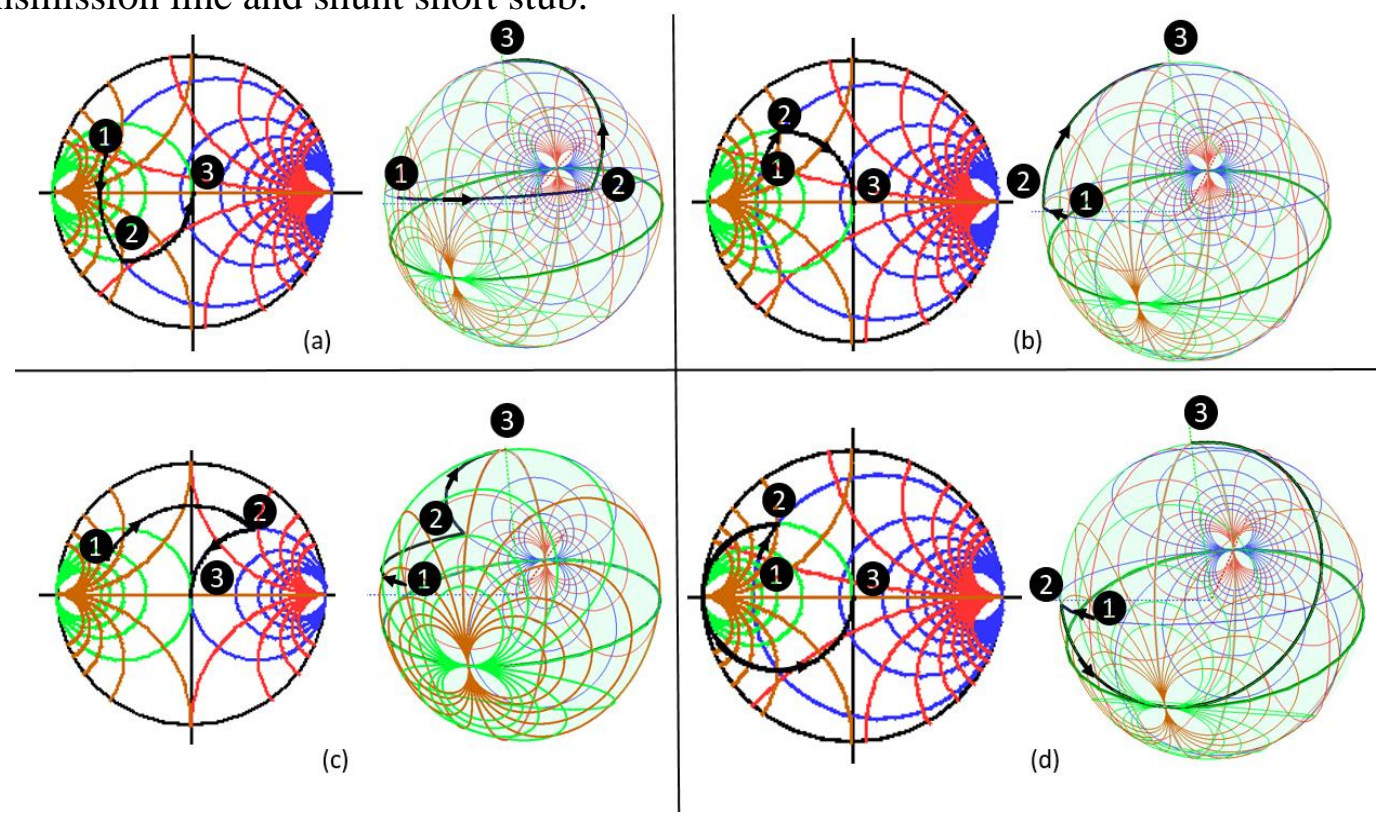

7 Fig. 7 Step-by-step matching of a 0.2+0.2j load using four different paths (a), (b), (c), and (d), on the Smith chart and on the 3D Smith chart 


\section{D space rendering above the 3D Smith chart used for both passive and active circuits}

Fig. 8 synthesizes the main rendering schemes proposed for displaying a variety of frequency-dependent parameters simultaneously with S-parameters, which are always displayed on the surface. Fig. 8 (a) shows the rendering which applies for the display of group delay and series and shunt inductance models. Mapping from the center of the sphere using variable homothety maps [15, 19] these values closer or further away from its surface (for each frequency point), corresponding to the normalized values displayed. If the values become negative, the values are mapped in the interior of the chart. Another positive scalar value that is dependent on the previous value is displayed as the variable radius of a generalized cylinder along the previously presented variable (Fig.8 (b)). Fig. 8 (c) presents the proposed frequency display as the distance from the corresponding $\mathrm{S}_{\mathrm{ij}}$ parameter (with a reference system in the center of the sphere).

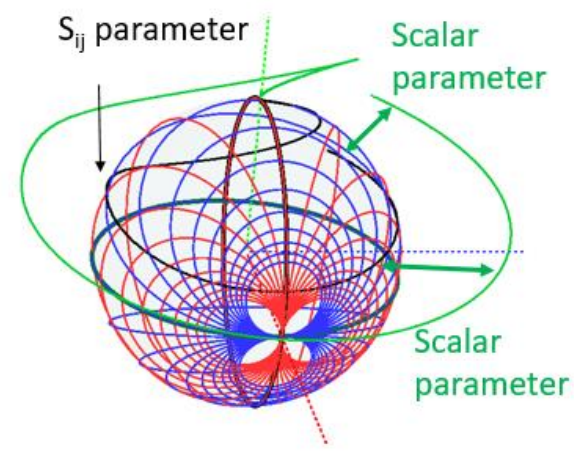

(a)

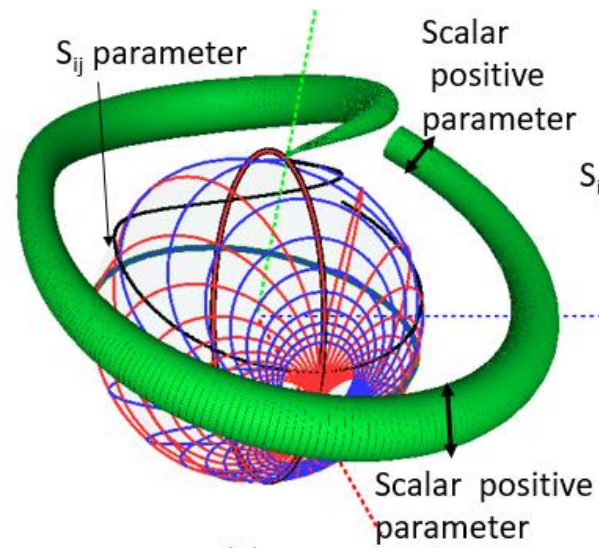

(b)

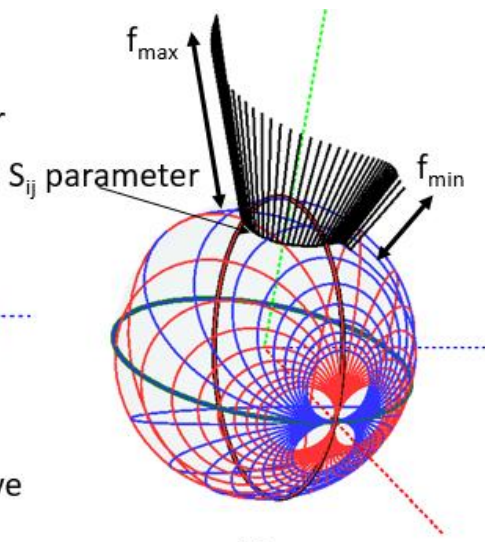

(c)

Fig. $83 D$ space representations of scalar values (a) parameter with possible positive values (exterior) and negative values (interior of the 3D chart) (group delay), (b) only positive values shown as a generalized cylinder with variable radius, and (c) frequency display graphing the dynamics of the S-parameters.

\section{Inductors: characterization of multiple simultaneous parameters}

The topology of the 3D Smith chart allows multiple parameter visualizations, which can potentially speed up the design/characterization of passive circuits, while giving new insights:

The authors of [19] report reconfigurable inductors with the phase change material vanadium dioxide $\left(\mathrm{VO}_{2}\right)$ as the switching material between insulating and conductive states with temperature control. Such programmable inductors outperform the inductors reported in [20] and [21], which use the same material, in terms of $\mathrm{Q}$ in the conductive state of $\mathrm{VO}_{2}$. The $\mathrm{VO}_{2}$ switch length of less than $635 \mathrm{~nm}$ minimizes its limited conductivity effects in [20], and quality factors of around 7 were achieved (unlike 3 in [20] or below unity in [21]) while using $\mathrm{VO}_{2}$.

Here we present other inductors with $1.6 \mathrm{um}$ switch length and their corresponding performances in on/off states measured with the ANRITSU Vector Star VNA and extracted with its incorporated software (Microwave Office) and our GUI. The results reported in Fig. 9 improve upon the results reported in [19] in terms of insulating state (off state) performance while performing less well in the on state (since the 1.6 um length of the $\mathrm{VO}_{2}$ switch accentuates the on-state losses). Fig. 9 (a) shows the layout of the inductor, which is the same as in [19] (but with a larger switch); (b) shows the measurement setup, including a heater to activate the $\mathrm{VO}_{2}$. The series inductance model (based on the $Y_{21}$ admittance parameter) [20-21] is shown in Fig. 9 (c); the shunt inductance model is shown in Fig. 9 (d) [22] (based on the $Y_{11}$ parameters); while $\mathrm{Q}$ is displayed in Fig. 9 (e). All of these are displayed on both a 2D plot and a 3D Smith chart together with the corresponding $S_{113 D}(j \omega)=\rho_{3 D}(j \omega)$ parameters.

The 3D representations are based on Fig. 9 (a) and Fig. 9 (b): 
For the 3D representation of the series and shunt inductance, the normalized $\left(L_{N}\right)$ values are first computed on the given frequency range of the display. The $\rho_{3 D}(2)$ is used and at each frequency point the 3D inductance curve is computed using the following formula:

$$
L_{3 d}(\omega)=\left(L_{N}(\omega)+1\right) * \rho_{3 D}(j \omega)
$$

In Fig 8 (c) - (e) multiple touchstone files are represented, then the maximum of the series and shunt inductance are used in the normalization step to obtain the normalized values across the different states displayed. The quality factor $\mathrm{Q}_{3 \mathrm{D}}$ is displayed as a generalized cylinder along the $L_{3 d}(\omega)$ curve with the variable radius corresponding to its value at the given frequency point normalized through its maximum over the display range [19]. When this becomes negative, the cylinder radius becomes 0 .

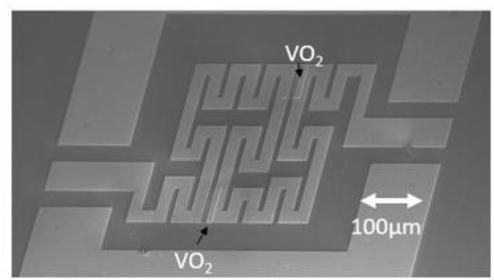

(a)

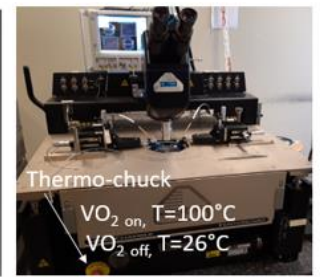

(b)

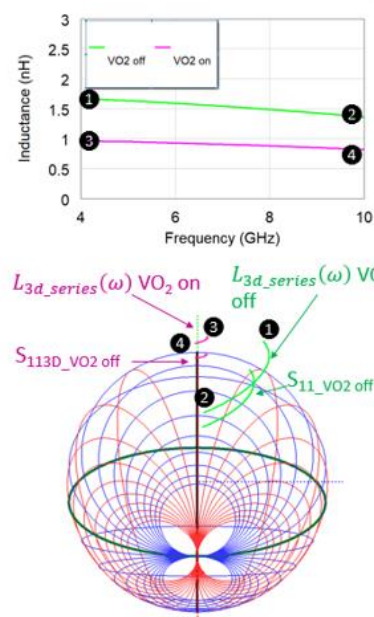

(c)

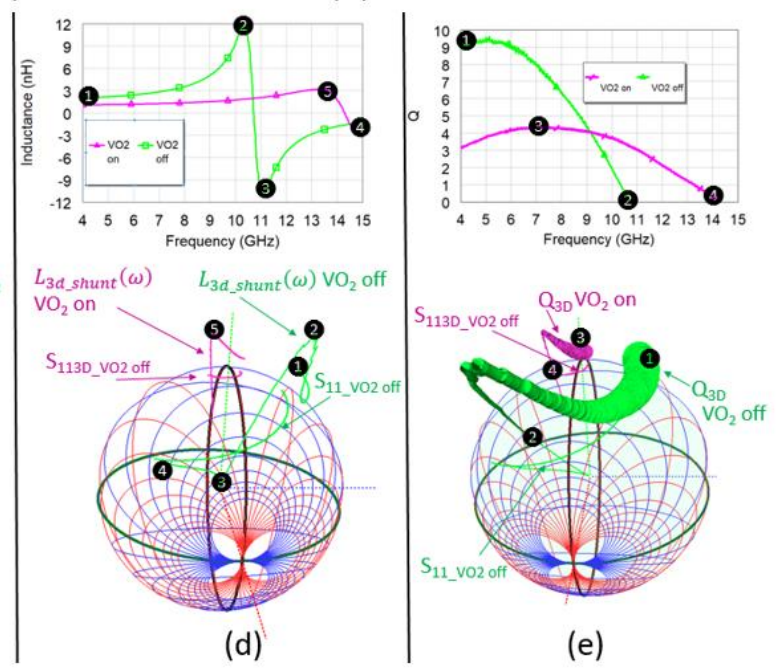

Fig. 9 (a) Inductor with $\mathrm{VO}_{2}$ switches, fabricated photo. (b) S-parameter measuring setup with heater for $\mathrm{VO}_{2}$ on/off activation. (c) Series inductance display over the S-parameters (d) Shunt inductance display over the S-parameters, (e) $Q$ factor display over the shunt inductance curve.

\section{Stability circles - inversive transformations}

The stability of an amplifier, or its resistance to oscillation, is a very important consideration in its design and can be determined from the S-parameters, matching networks, and terminations.

Some passive loads and source terminations can produce input and output impedances that have negative real parts; oscillations are possible under these circumstances. A designer would aim for unconditional stability: that is, for possible loads and source terminations, his/her amplifier should not produce negative real parts of its input and output impedance.

A graphical way to check this, and thus to ensure unconditional stability, is to ensure that the load (input) stability and the source (output) stability circles are mapped in the exterior of the Smith chart (both their centers and all their points) $[16,23]$. The positions of these circles are given by (5) (a) and (5) (b) where $\mathrm{z}$ denotes the unit circle and $\Delta$ the determinant of the two-port S-parameters [23] (7).

Writing (5) as (6) we can see that we have an inversive transformation of $\mathrm{z}$, where $\mathrm{z}$ is the unit circle. Geometrically, for 5(b): step a) translation of the unit circle with $\frac{\Delta}{s_{22}}$, step $b$ ) inversion in the unit circle, step c) dilatation with $\operatorname{abs}\left(\frac{S_{12} S_{21}}{S_{22}^{2}}\right)$, step $\left.d\right)$ rotation with $\arg \left(\frac{S_{12} S_{21}}{S_{22}^{2}}\right)$

$$
t(z)=\frac{z-S_{11}}{S_{22} z-\Delta} \text { (a) } \quad p(z)=\frac{S_{22}-z}{\Delta-S_{11} z}
$$




$$
\begin{gathered}
t(z)=\frac{1}{S_{22}}-\frac{\frac{s_{12} S_{21}}{S_{22}^{2}}}{z-\frac{\Delta}{S_{22}}} \quad \text { (a) } \quad p(z)=\frac{1}{S_{11}}+\frac{\frac{S_{21} S_{12}}{S_{22}^{2}}}{z-\frac{\Delta}{S_{11}}} \text { (b) } \\
\Delta=S_{11} * S_{22}-S_{21} * S_{12}
\end{gathered}
$$

In the rather unusual but possible situation that $\left|\frac{\Delta}{s_{22}}\right|=1$ step a) will translate the unit circle in 5 (b) into a circle passing through the origin in a point. Step $b$ ) will then map this circle into an extended line passing through infinity (since inversions in circles passing through the origin result in infinite lines), steps $c$ ) and d) will not change this any further, resulting in an extended line. The same things occur with 6(b) for $\left|\frac{\Delta}{s_{11}}\right|=1$; thus, theoretically, one can deal with circles or extended lines within the 2D complex reflection coefficients plane for (5) and (6).

On the 3D Smith chart there are no exceptions; (5) and (6) are inversive transformations of the unit circle, if $\left|\frac{\Delta}{S_{22}}\right|=1$ or $\left|\frac{\Delta}{S_{11}}\right|=1$ both (5) and (6) will generate circles passing through the south pole.

In order to ease the use of stability circles once their centers are mapped toward infinity (and thus become impossible to visualize on a 2D Smith chart): we propose the use of the 3D Smith chart for checking the stability in a compact and general way.

\section{Example 2: Stabilize a transistor with a shunt resistor}

The following example shows how a resistive loading can stabilize a potentially unstable transistor using the graphical methodology presented above. In order to test it, we will also compute the values of classical stability coefficients in both situations.

Determine the resistive load that can stabilize the transistor with the following S-parameters presented in Table V at $800 \mathrm{MHz}$ [16, pp. 227] and plot the stability circles in both situations.

The stability circles can be seen in Fig. 10 (a) and (b) on the 3D Smith chart. In order to see the values needed for $r$ and $g$ to stabilize the input stability circle (black) we change the rendering for $r, x$ and $g, b$ and detect the values needed.

Table IV: S-parameters of the Given Transistor

\begin{tabular}{|l|l|l|l|l|l|l|l|}
\hline$\left|\mathrm{S}_{11}\right|$ & phase $^{\mathrm{o}}$ & $\left|\mathrm{S}_{12}\right|$ & phase & $\left|\mathrm{S}_{21}\right|$ & phase & $\left|\mathrm{S}_{22}\right|$ & phase \\
\hline 0.65 & -95 & 0.035 & 40 & 5 & 115 & 0.8 & -35 \\
\hline
\end{tabular}

Stability tests for the transistor presented in Table IV based on the Rollet (8) equation [16, 24, 25] show $k=0.54$ with $|\Delta|=0.50$, or the more compact versions [26] given in (9) result in $\mu_{1}=0.85$, thus providing a violation of the conditions.

$$
\begin{gathered}
k=\frac{1+|\Delta|^{2}-\left|S_{11}\right|^{2}-\left|S_{22}\right|^{2}}{2\left|S_{12} * S_{21}\right|}>1 \text { and }|\Delta|=<1 \\
\mu_{1}=\frac{1-\left|S_{11}\right|^{2}}{\left|S_{22}-S_{11}^{*} \Delta\right|+\left|S_{12} * S_{21}\right|}>1
\end{gathered}
$$

Moreover, since $k$ is smaller than unity, the stability circles will cross the boundary of the Smith chart [25], or the equator of the 3D Smith chart; our aim is to shift their centers and all their points outside of the northern hemisphere. Fig. 11 (a) and (b) shows the stability circles' positions once the transistor is stabilized-corresponding to the S-parameters in Table V. Adding a shunt resistor of $500 \Omega$, the new Sparameters can stabilize the transistor (schematic view in Fig. 11) exhibiting the S-parameters in Table V providing $k=1.04$ with $|\Delta|=0.40$, and $\mu_{1}=1.02$, which is thus unconditionally stable, according to (9).

Table V: S-parameters of the Given Transistor with a Shunt Resistor Connected 


\begin{tabular}{|l|l|l|l|l|l|l|l|}
\hline$\left|\mathbf{S}_{11}\right|$ & phase $^{\mathbf{0}}$ & $\left|\mathrm{S}_{12}\right|$ & phase $^{\mathbf{0}}$ & $\left|\mathbf{S}_{21}\right|$ & phase $^{\mathbf{0}}$ & $\left|\mathbf{S}_{22}\right|$ & phase $^{\mathbf{~}}$ \\
\hline 0.65 & -94 & 0.032 & 41.2 & 4.62 & 116.2 & 0.66 & -36 \\
\hline
\end{tabular}

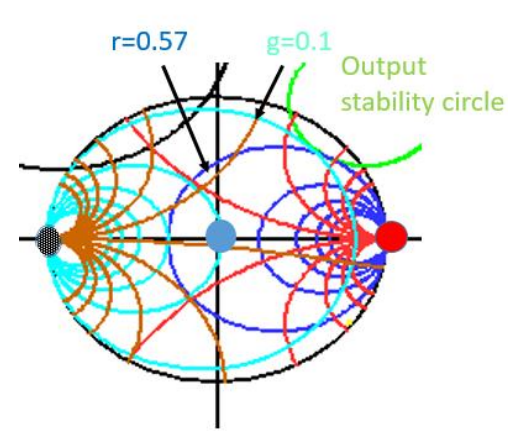

(a)

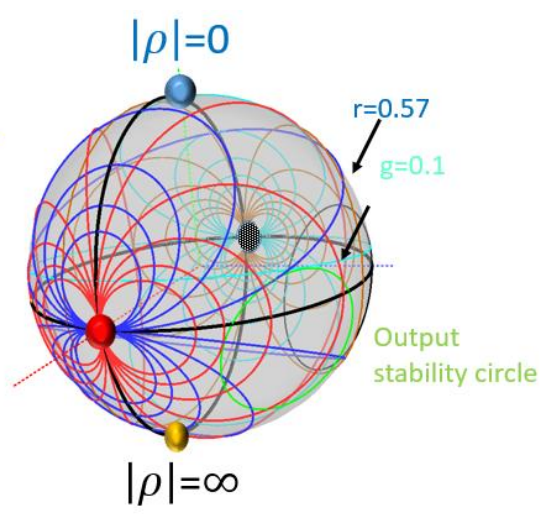

(b)

Fig.10 Stability circles for the transistor doscrihod in Tahlo V at 8OO MH7 (hlark innut oroon outnut) $($ a) Smith chart, (b) $3 D$ Smith chart.

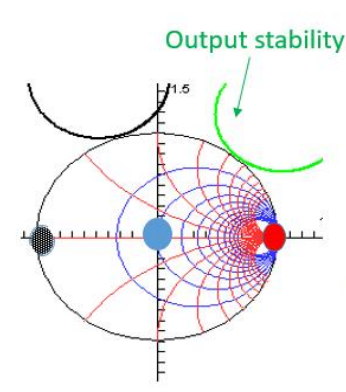

(a)

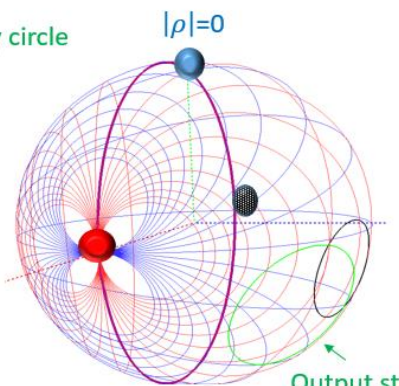

(b)

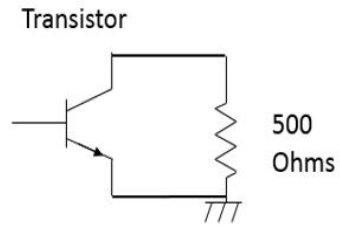

(c)

Fig. 11 Stabilized transistor with a $500 \Omega$ resistor: (a) Stability circles on the Smith chart, (b) 3D Smith chart, (c) Stabilization scheme

\section{Power gain circles - pure Euclidean geometry in 3D}

Unilateral transducer constant power gain circles play an essential role in the design of radio-frequency amplifiers and active modulators, as they help to determine optimal impedance matching conditions to meet gain and stability specifications. Unilateral transducer constant power gain circles (for the source input in our example) are a subfamily of Apollonius circles, with respect to $S_{11} *$ and $1 / S_{11}$. Plotting these gain circles on the 3D Smith chart overcomes traditional limits associated with contour plots on the traditional 2D Smith chart [14].

\section{Example 3:}

Consider the following BJT S-parameters measured at $1 \mathrm{GHz}$ [16], given in Table VI.

The maximum achievable gain is $10\left(1 / \mathrm{Lg}\left|1-S_{11}\right|^{2}\right)=3.31 \mathrm{~dB}$

Table VI: BJT S-parameters at $1 \mathrm{GHz}$

\begin{tabular}{|c|l|l|l|l|l|l|l|}
\hline$\left|S_{11}\right|$ & Phase $^{\circ}$ & $\left|S_{12}\right|$ & Phase $^{\circ}$ & $\left|S_{21}\right|$ & Phase $^{\circ}$ & $\left|S_{22}\right|$ & Phase $^{\circ}$ \\
\hline 0.73 & $175^{\circ}$ & 0 & 0 & 4.45 & 65 & 0.21 & $-80^{\circ}$ \\
\hline
\end{tabular}

Plot the $3.31 \mathrm{~dB}$ constant power gain circles and the -2.27 power gain circles.

In Fig. 12 we plot the gain circles corresponding to Table VII, which is the min/max values computed for the BJT transistor given in Table VI. The power level in 3D is normalized with respect to the square root of the maximum gain (for display reasons).

Table VII: BJT S-parameters at $1 \mathrm{GHz}$ 


\begin{tabular}{|l|l|l|}
\hline $\begin{array}{l}\text { Gain } \\
\mathrm{dB}\end{array}$ & Gain & Gain^.5 \\
\hline 3.31 & 2.15 & 1.46 \\
\hline 1.78 & 1.51 & 1.23 \\
\hline 0 & 1 & 1 \\
\hline-2.27 & 0.6 & 0.77 \\
\hline
\end{tabular}

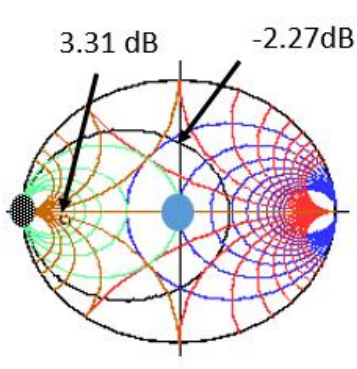

(a)

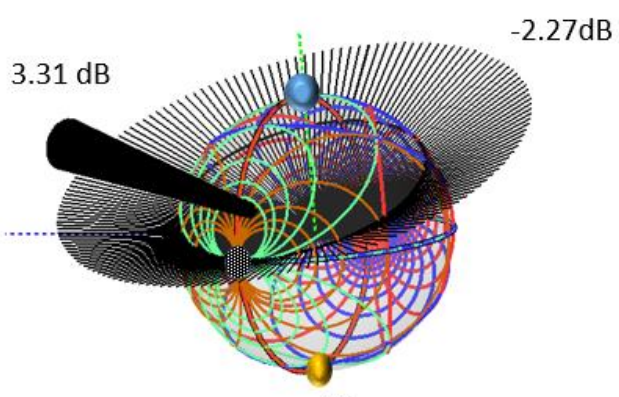

(b)

Fig. 12 Power gain circles for two different power levels (a) on a 2D Smith chart and (b) on a 3D Smith chart

\section{Example 4: Negative capacitance}

Negative capacitance [26-30] is of interest in many RF applications; however, the negative capacitance and positive inductance $S_{11}$ parameters of 1-port and 2-port lossless networks share the same paths on the Smith chart. By means of differential geometry, however, we can see that their orientation (oriented curvature [20] of their frequency $\mathrm{S}_{11}$ ) in either 1- or 2-port configurations is opposed. Their Foster/nonFoster character [27-30] changes the orientation of their $S_{11}$ motions as frequency increases on the Smith chart.

Fig.13 (a) represents the $S_{11}$ of a negative capacitor and positive inductor in a 1-port configuration with a $50 \Omega$ load at both ports analyzed between 1-7 GHz. The implementation in [19] distinguishes the different intrinsic natures (opposed sign of oriented curvatures) of both by representing the sweeping frequency dependency in 3D as in Fig. 13 (b). In this representation we can detect not only the path, but also the dynamics of the motion. The frequency representation in [19] allows the quantitative display of the frequency in $3 \mathrm{D}$ as the distance from the 3D Smith chart for each $S_{11}$ point. The counterclockwise frequency increase motion is clearly detected in Fig. 13 (b) for the negative capacitance, thus proving its different nature with respect to a conventional positive inductor. Even though its $S_{11}$ parameters coincide on the Smith chart on a large frequency range with the $S_{11}$ parameters of a conventional positive inductor, Fig. 13 (b) detects the different intrinsic nature of the negative capacitance.

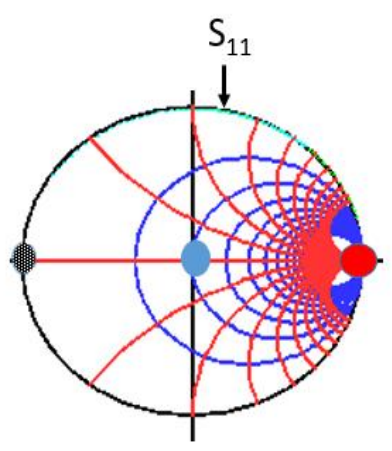

(a)

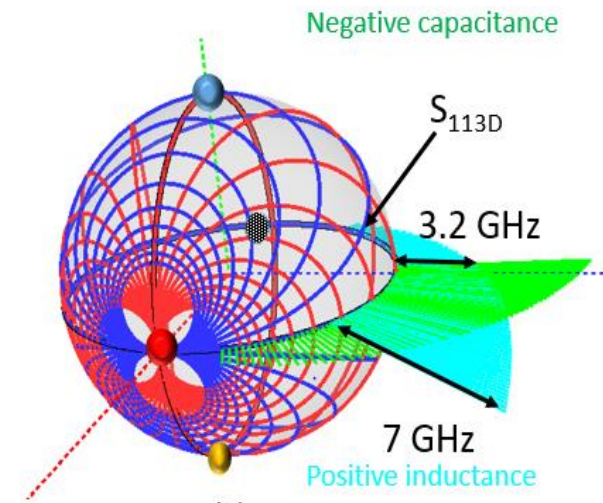

(b)

Fig. 13 Negative capacitance and positive inductance reflection coefficients on (a) 2D Smith chart, (b) Frequency dependent 3D Smith chart 


\section{Example 5: Negative resistance circuits}

Let us consider a negative resistance phenomenon [31], such as the one observed in tunnel diodes [32] (Esaki diodes), which have been employed in active circuit design since 1957. Fig. 14 (a) depicts a small signal equivalent circuit [4] consisting of a negative resistance $R$, unavoidable shunt capacitance $C$, series inductance $L$, and series resistance $R_{s}$. With the additional presence of a very lossy package, including the presence of additional parasitic [33] $R_{p}, L_{p}$, and $C_{p}$, and taking into account the values depicted in Fig. 14, compute the input impedance $\left(Z_{i n}\right)$ at $2 \mathrm{GHz}$.

Fig. 14 (b) depicts the computation procedure using the 3D Smith chart, by means of normalized values to $50 \Omega(0.02 \mathrm{~S})$. The input normalized impedance detected with the 3D Smith chart is $z i n=1 /(\mathrm{g}+\mathrm{jb}) \simeq 0.037$ $0.147 \mathrm{j}$, thus $\mathrm{Z}_{\mathrm{in}} \simeq-1.85-\mathrm{j} 7.35$.
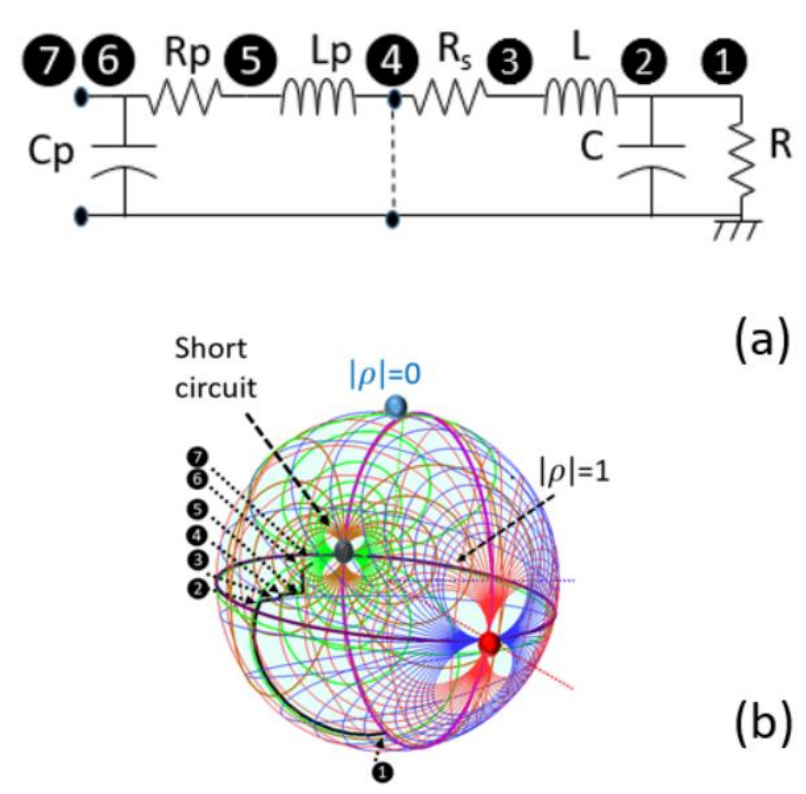

(a)

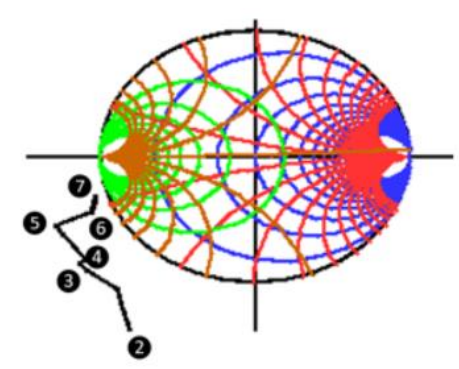

(b)
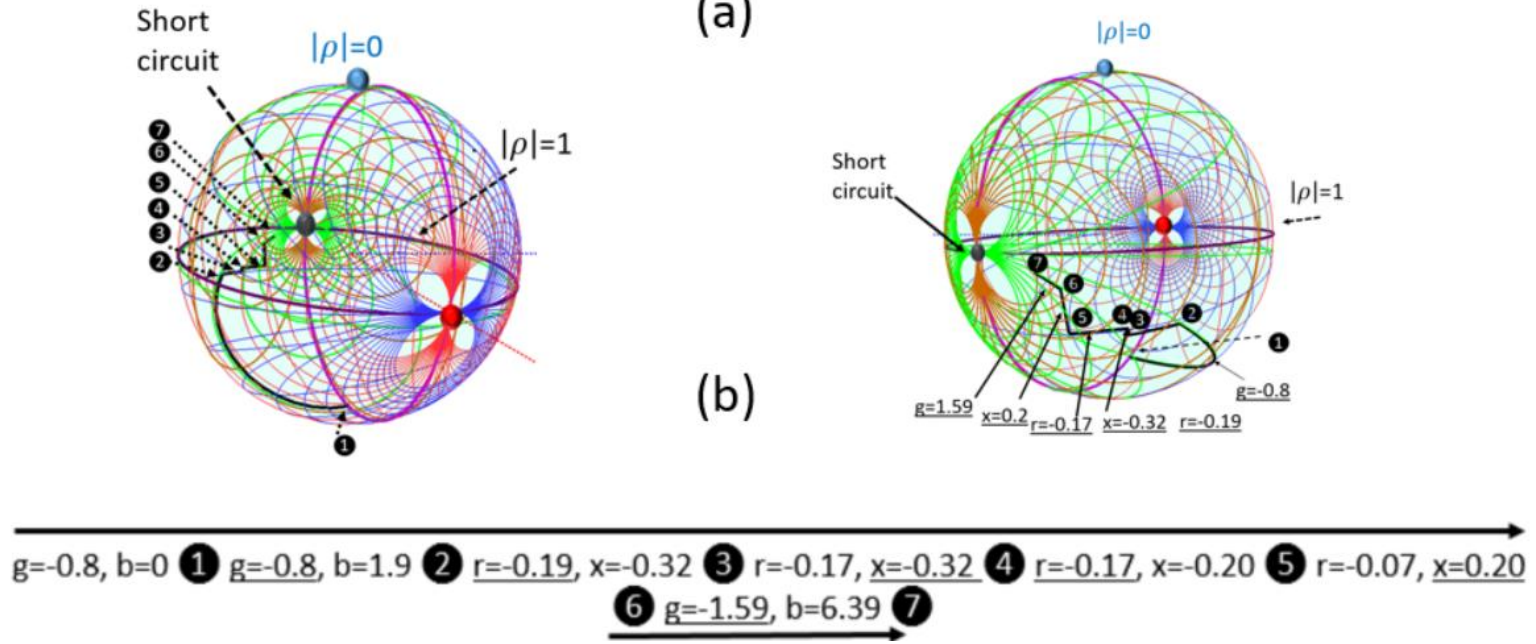

Fig. 14 Tunnel diode equivalent circuit, including the packaging at $2 \mathrm{GHz}$ with $(a) . R=-62.5 \Omega(g=-0.8), C=3 \mathrm{pF}(b=1.9)$, $L=0.5 \mathrm{nH}(x=0.126), R_{S}=1 \Omega(r=0.02), L_{p}=0.5 \mathrm{nH}(x=0.126), R_{p}=5 \Omega(x=0.1), C_{p}=3 \mathrm{pF}(b=1.9)$, and its Smith chart impedance in different points. (b) $3 D$ Smith chart impedance computation seen from two perspectives

\section{Conclusions}

This paper has reported on the historical evolution of the Smith chart and its current extension and analytical generalization as a $3 \mathrm{D}$ tool.

We have illustrated the theoretical development, refinement, and capabilities of the 3D Smith chart with a series of examples from passive to active components, including concepts such as negative resistance and negative capacitance circuits. For all selected examples we used the same basic mathematical formulations and we have reported how inversive-differential geometry theory can be used to develop an engineering 
tool that enhances the visualization capability of traditional Smith charts and offers new insights into the design of radio frequency devices and circuits.

ACK: Parts of this work were supported by the HORIZON2020 FETOPEN PHASE-CHANGE SWITCH Project under Grant 737109 and the PGC2018-094889-B-100 grant. The authors would like to specially thank to Prof. Robert Caverly, assistant editor Sharri Shaw and the Editorial board \& reviewers for the very helpful suggestions, constructive critics and corrections throughout the entire review process. Last, we would like to thank Prof $\mathrm{Ke} \mathrm{Wu}$ for his idea to encourage the authors in writing an article on this topic.

\section{References}

[1] R. W. Rhea, Preface of "Electronic Applications of the Smith chart", $2^{\text {nd }}$ ed. Noble publishing, 2000.

[2] P. H. Smith, “Transmission-line calculator”, Electronics, vol. 12, pp. 29-31, Jan. 1939.

[3] P. H. Smith, “An improved transmission line calculator”, Electronics, Jan. 1944.

[4] P. H. Smith, "Electronic Applications of the Smith chart", McGraw Hill Book Company, New York 1969.

[5] M. Bocher, "Infinite Regions of Various Geometries', Bull. Amer. Math. Soc., 20, 4,185-200 (1914).

[6] R.L. Kyhl, "Plotting Impedances with Negative Resistance Components", IEEE Trans. G-MTT, vol.MTT-8, no. 3, p.377, May 1960.

[7] S. Gupta, "Escher's art, Smith Chart and hyperbolic geometry", IEEE Microwave Magazine, vol. 7, pp. 67-76, Oct. 2006.

[8] C. Zelley, "A spherical representation of the Smith Chart," IEEE Microwave Magazine, vol. 8, pp. 6066, Jun. 2007.

[9] Y. Wu, H. Huang, Y. Liu and Z. Gao, "Spherical representation of the omnipotent Smith chart", Microw. Opt. Technol. Lett., vol. 50, no. 9, pp. 2452-2455, Sep. 2008.

[10] Y.Wu, Y. Zhang, Y. Liu, and H. Huang, "Theory of the spherical generalized Smith Chart," Microw. Opt. Technol. Lett., vol. 51, pp. 95-97, Jan. 2009.

[11] D. A. Brannan, M.F. Esplen, J.J. Gray, Geometry. Cambridge University Press, New York (2007).

[12] A. A. Muller, P. Soto, D. Dascalu, D. Neculoiu and V. E. Boria, "A 3D Smith Chart based on the Riemann Sphere for Active and Passive Microwave Circuits," IEEE Microw. Compon. Lett., vol. 21, no. 6, pp. 286-288, June 2011.

[13] A. A. Muller, P. Soto, D. Dascalu, and V. E. Boria, "The Practical Applications of the 3D Smith chart”, Microwave Journal, vol. 55, no. 7, pp. 64-72, July 2012.

[14] A. A. Muller, E. Sanabria-Codesal, P. Soto, A. Moldoveanu, V. Asavei, V. E. Boria and S. Lucyszyn, "Apollonius unilateral transducer constant power gain circles on the 3D Smith chart," IET Electron. Lett., vol. 50, no. 21, pp. 1531-1533, Oct. 2014.

[15] A. A Muller, E. Sanabria-Codesal, A. Moldoveanu, V. Asavei and S. Lucyszyn "Extended Capabilities of the 3D Smith chart with group delay and resonator quality factor" IEEE Trans. Microw. Theory Tech., vol. 65, No. 1. pp. 10-19, Jan. 2017.

[16] G. Gonzalez, "Microwave Transistor Amplifiers Analysis and design", Prentice Hall, $2^{\text {nd }}$ edition, 1996.

[17] B. C. Wadell “Smith Charts Are Easy-Part 1”, IEEE Instrum. Meas. Mag., vol.2,1, 37-40, 1999.

[18] B. C. Wadell “Smith Charts Are Easy-Part 2", IEEE Instrum. Meas. Mag., vol.2,2, 45-47, 1999.

[19] A. A. Muller et al., 3D Smith charts scattering parameters frequency-dependent orientation analysis and complex-scalar multi-parameter characterization applied to Peano reconfigurable vanadium dioxide inductors, Nat. Scientific Reports, 9, 18346, Dec. 2019, pp 1-15. 
[20] E. A. Casu et al., A reconfigurable inductor based on Vanadium Dioxide insulator to metal transition. IEEE Microw. Compon. Lett. 29, 9, 795-797 (2018).

[21] S. Wang et al., Tunable inductors using vanadium dioxide as the control material. Microw. Opt. Techn. Lett., 59, 5, 1057-1061 2017.

[22] J. Kang et al., On-chip intercalated-graphene inductors for next-generation radio frequency electronics. Nature Electronics, 1 46-51, 2018.

[23] J. F. White, High Frequency Techniques: An Introduction to RF and Microwave Engineering, Hoboken, NJ: John Wiley \& Sons, 2004

[24] M. J. Rollet "Stability and Power Gain Invariants of the Linear Two Ports", IRE Transactions on Circuit Theory, vol ct 9, pp 29-32, March 1962

[25] M. L. Edwards, J. H. Sinsky, “A New Criterion for Linear Two Port Stability Using a Single Geometrical Dervied Parameter, IEEE Trans. Microw. Theory Tech,vol 40, no 12, pp 2303-2310, Dec 1992

[26] A. M. Ionescu, "Negative capacitance gives a positive boost", Nat. Nanotechnol. 13, 7-8 (2018).

[27] H. Mirzaei and G. V. Eleftheriades, "Realizing non-Foster reactive elements using negative-group delay networks", IEEE Trans. Microw. Theory Tech., vol. 61, no. 12, pp. 4322-4332, Dec. 2013

[28] A. A. Muller and S. Lucyszyn, "Properties of purely reactive Foster and Non-Foster passive networks", IET Electron. Lett., vol. 51, no. 23, pp. 1882-1884, Nov. 2015.

[29] A. M. Kemp, et al., A high Q piezoelectric resonator as a portable VLF transmitter. Nat. Commun., $10,17152019$.

[30] M. M. Jacob, Non-Foster circuits for high performance antennas: advantages and practical limitations. In PhD thesis San Diego UCSD (2016), https://escholarship.org/uc/item/2pq549c3.

[31] D. Dascalu Transit-time effects in unipolar solid-state devices Abacus Press, Tunbridge Wells, Kent, England (1972)

[32] A. M. Ionescu, H. Riel, "Tunnel field-effect transistors as energy-efficient electronic switches", Nature, 479, pp 329-337, 2011.

[33] K. Ishii, Practical Microwave Electron Devices, Academic Press Inc., San Diego, CA, 1990. 